\title{
Alignment-Free Phylogenetic Reconstruction
}

\author{
Constantinos Daskalakis* $\quad$ Sebastien Roch ${ }^{\dagger}$
}

October 6, 2009

\begin{abstract}
We introduce the first polynomial-time phylogenetic reconstruction algorithm under a model of sequence evolution allowing insertions and deletions-or indels. Given appropriate assumptions, our algorithm requires sequence lengths growing polynomially in the number of leaf taxa. Our techniques are distance-based and largely bypass the problem of multiple alignment.
\end{abstract}

${ }^{*}$ CSAIL, MIT.

${ }^{\dagger}$ Department of Mathematics and Bioinformatics Progam, UCLA. 


\section{Introduction}

We introduce a new efficient algorithm for the phylogenetic tree reconstruction (PTR) problem which rigorously accounts for insertions and deletions.

Phylogenetic background. A phylogenetic tree or phylogeny is a tree representing the speciation history of a group of organisms. The leaves of the tree are typically existing species. The root corresponds to their most recent common ancestor (MRCA). Each branching in the tree indicates a speciation event. It is common to assume that DNA evolves according to a Markovian substitution process on this phylogeny. Under such a model, a gene is a sequence in $\{\mathrm{A}, \mathrm{G}, \mathrm{C}, \mathrm{T}\}^{k}$. Along each edge of the tree, each site independently mutates according to a Markov rate matrix. The length of a branch is a measure of the amount of substitution along that branch. ${ }^{1}$ The PTR problem consists in estimating a phylogeny from the genes observed at its leaves. We denote the leaves of a tree by $[n]=\{1, \ldots, n\}$ and their sequences by $\sigma_{1}, \ldots, \sigma_{n}$.

The model of sequence evolution above is simplistic: it ignores many mutational events that DNA undergoes through evolution. At the gene level, the most important omissions are insertions and deletions of sites, also called indels. Stochastic models taking indels into account have long been known [TKF91, TKF92], but they are not widely used in practice—or in theory—because of their complexity. Instead, most practical algorithms take a two-phase approach:

1. Multiple sequence alignment. Site $t_{i}$ of sequence $\sigma_{i}$ and site $t_{j}$ of sequence $\sigma_{j}$ are said to be homologous if they descend from the same site $t_{0}$ of a common ancestor $u$ (not necessarily the MRCA) only through substitutions. In the multiple sequence alignment (MSA) problem, we seek roughly to uncover the homology relation between $\sigma_{1}, \ldots, \sigma_{n}$. Typically, the output is represented by a matrix $\mathbf{D}$ of $n$ aligned sequences of equal length with values in $\{\mathrm{A}, \mathrm{G}, \mathrm{C}, \mathrm{T},-\}$. Each column of the matrix corresponds to homologous sites. The state - is called a gap and is used to account for insertions and deletions. For instance if sequence $\sigma_{l}$ does not have a site corresponding to $t_{0}$ in $u$ above, then a gap is aligned with positions $t_{i}$ of $\sigma_{i}$ and $t_{j}$ of $\sigma_{j}$ (which belong to the same column).

2. Phylogenetic tree reconstruction. The matrix $\mathbf{D}$ is then cleaned up by removing all columns containing gaps. Let $\mathbf{D}^{\prime}$ be this new matrix. A standard PTR algorithm is then applied to $\mathbf{D}^{\prime}$. Note that substitutions alone suffice to explain $\mathbf{D}^{\prime}$.

Traditionally, most of the research on phylogenetic methods has focused on the second phase.

In fact, current theoretical analyses of PTR assume that the MSA problem has been solved perfectly. This has been a long-standing assumption in evolutionary biology. But this simplification is increasingly being questioned in the phylogenetic literature, where it has been argued that alignment heuristics often create systematic biases that affect analysis [LG08, WSH08]. Much recent empirical work has been devoted to the proper joint estimation of alignments and phylogenies [TKF91, TKF92, Met03, MLH04, SR06, RE08, LG08, $\mathrm{LRN}^{+}$09]. Here, we give the first analysis of an efficient, provably consistent PTR algorithm in the presence of indels. Our new algorithm suggests that a rough alignment suffices for an accurate tree reconstruction—bypassing the computationally difficult multiple alignment problem.

Theoretical properties of PTR. In addition to computational efficiency, an important theoretical criterion in designing a PTR algorithm is the so-called sequence-length requirement (SLR). At a minimum, a reconstruction algorithm should be consistent, that is, assuming a model of sequence evolution, the output should be guaranteed to converge on the true tree as the sequence length $k$ - the number of samples - goes to

\footnotetext{
${ }^{1}$ The precise definition of a branch length depends on the model of evolution. For roughly constant mutation rates, one can think of the branch length as proportional to the amount of time elapsed along a branch.
} 
$+\infty$ [Fel78]. Beyond consistency, the sequence-length requirement-or convergence rate- of a PTR algorithm is the sequence length required for guaranteed high-probability reconstruction. The SLR is typically given as an asymptotic function of $n$, the number of leaves of the tree. Of course, it also depends on the substitution parameters.

A classical result due to Erdös et al. [ESSW99a] states that, for general trees under the assumption that all branch lengths are bounded by constants, the so-called Short Quartet Method (SQM) has poly $(n)$-SLR. The SQM is a particular PTR algorithm based on estimating evolutionary distances between the leaf taxa, that is, the sum of the branch lengths between species. Such algorithms are known as distance-based methods. The basic theoretical result behind distance-based methods is the following: the collection of pairwise evolutionary distances between all species forms a special metric on the leaves known as an additive metric; under mild regularity assumptions, such a metric characterizes the underlying phylogeny interpreted as an edge-weighted tree, that is, there is a one-to-one correspondence between additive metrics and phylogenies; moreover, the mapping between them can be computed efficiently [SS03].

A new approach. In the classical theoretical setting above where the MSA problem is assumed perfectly solved (we refer to this setting below as the ESSW framework), the evolutionary distance between two species is measured using the Hamming distance (or a state-dependent generalization) between their respective sequences. It can be shown that after a proper correction for multiple substitutions (which depends on the model used) the expectation of the quantity obtained does satisfy the additive metric property and can therefore serve as the basis for a distance-based PTR algorithm.

Moving beyond the ESSW framework, it is tempting to account for indels by simply using edit distance instead of the Hamming distance. Recall that the edit distance or Levenshtein distance between two strings is given by the minimum number of operations needed to transform one string into the other, where an operation is an insertion, deletion, or substitution of a single character. However, no analytical expression is known for the expectation of edit distance under standard indel models and computing such an expression appears difficult-if at all possible. An alternative idea is to compute the maximum likelihood estimator for the time elapsed between two species given their sequences. But this involves solving a nonconvex optimization problem and the likelihood is only known to be efficiently computable under a rather unrealistic assumption known as reversibility [TKF91] (see below).

We use a different approach. We divide the sequences into quantile blocks (the first $x \%$, the second $x \%$, etc.). We show that by appropriately choosing $x$ above we can make sure that the blocks in different sequences essentially "match" each other, that is, they are made of mostly homologous sites. We then compare the state frequencies in matching blocks and build an additive metric out of this statistic. As we show below, this is in fact a natural generalization of the Hamming estimator of the ESSW framework. However, unlike the Hamming distance which can easily be analyzed through standard concentration inequalities, proving rigorously that our approach works involves several new technical difficulties. We give a quick proof sketch after the formal statement of our results in Section 1.2.

Related work. For more background on models of molecular evolution and phylogenetics, see, e.g., [GL99, SS03, Fel04]. Following the seminal results of [ESSW99a], there has been much work on sequence-length requirement, including [Att99, ESSW99b, HNW99, SS99, CK01, Csu02, SS02, KZZ03, MR06, DMR06, LC06, $\mathrm{DHJ}^{+}$06, Mos07, GMS08, Roc08, DMR09].

The multiple sequence alignment problem as a combinatorial optimization problem (finding the best alignment under a given pairwise scoring function) is known to be NP-hard [WJ94, Eli06]. Most heuristics used in practice, such as CLUSTAL [HS88], MAFFT [KMKM02], and MUSCLE [Edg04], use the idea of a guide tree, that is, they first construct a very rough phylogenetic tree from the data (using for instance edit distance as a measure of evolutionary distance), and then recursively construct local alignments produced 
by "aligning alignments."

To our knowledge, little theoretical work has been dedicated to the joint estimation of alignments and phylogenies, with the exception of Thatte [Tha06] who gave consistency results for the reversible case in the limit where the deletion-to-insertion ratio tends to 1 . However, no sequence-length requirement is obtained in [Tha06]. In recent related work, Andoni et al. [ADHR09] considered the problem of reconstructing ancestral sequences in the presence of indels.

\subsection{Model of Sequence Evolution}

Phylogeny. A phylogeny is represented by a binary tree $T=(V, E)$, whose leaves $L \subset V$ correspond to extant species, and whose bifurcations denote evolutionary events whereby two new species are generated from an ancestor. The root of the phylogeny, denoted by $r(T)$, represents the common ancestor of all the species in the phylogeny, and we assume that all edges of $T$ are directed away from $r(T)$; so, if $e=(u, v)$ is a branch of the phylogeny, $u$ is the parent of $v$ and $v$ is the child of $u$. Moreover, if $v^{\prime}$ is in the subtree of $T$ rooted at $u$, we call $v^{\prime}$ a descendant of $u$ and $u$ an ancestor of $v^{\prime}$.

Along each branch of the phylogeny, the genetic material of the parent species is subject to modifications that produce the genetic material of its child species. A common biological assumption is that the genetic material of each species $u$ can be represented by a binary sequence $\sigma_{u}=\left(\sigma_{u}^{1}, \ldots, \sigma_{u}^{K_{u}}\right)$ of length $K_{u}$ over a finite alphabet—we work here with the binary alphabet $\{0,1\}$ for simplicity ${ }^{2}$-and that the changes to which $\sigma_{u}$ is subjected along the branch $e=(u, v)$ are described by a Markov process. In particular, the Markov property implies that, given the sequence $\sigma_{u}$ of $u$, the sequence $\sigma_{v}$ is independent of the sequences of the species outside the subtree of $T$ rooted at $u$.

A simplifying assumption commonly used in phylogenetics is that all species have sequences of the same length and, moreover, that every site, i.e., every coordinate, in their sequences evolves independently from every other site. In particular, it is assumed that, along each branch $e=(u, v)$ of the phylogeny, every site $\sigma_{u}^{j}$ of the sequence $\sigma_{u}$ is flipped with probability $p_{e}$ to the value $1-\sigma_{u}^{j}$ independently from the other sites. This model is known as the Cavender-Farris-Neyman (CFN) model. A simple generalization to $\{$ A, G, C, T $\}$ is known as the Jukes-Cantor (JC) model. See, e.g., [Fel04].

Accouting for indels. In this paper, we consider a more general evolutionary process that accounts for the possibility of insertions and deletions. Our model is similar to the original TKF91 model [TKF91], except that we do not enforce reversibility. ${ }^{3}$ In our model, every edge $e=(u, v)$ of the phylogeny is characterized by a quadruple of parameters $\left(t_{e} ; \eta_{e}, \mu_{e}, \lambda_{e}\right)$, where $t_{e}$ is the evolutionary time between the species $u$ and $v$, and $\eta_{e}, \mu_{e}$ and $\lambda_{e}$ are respectively the substitution, deletion and insertion rates. The process by which the sequence at $v$ is obtained from the sequence at $u$ is defined below. (The process can be simply described as a continuous-time Markov process [KT81]. We give a full description for clarity.)

Definition 1.1 (Evolutionary Process on a Branch) Given an edge $e=(u, v)$, with parameters $\left(t_{e} ; \eta_{e}, \mu_{e}, \lambda_{e}\right)$, the sequence $\sigma_{v}$ at $v$ is obtained from the sequence $\sigma_{u}$ at $u$ according to the following Markov procedure:

1. intialize $\sigma_{v}:=\sigma_{u}, K_{v}:=K_{u}$ and $t_{\ell}:=t_{e}$; $/ * t_{\ell}$ is the remaining time on the edge $e * /$

2. while $t_{\ell}>0$

\footnotetext{
${ }^{2}$ We can also consider richer alphabets, e.g., $\{$ A $, C, G, T\}$, without much modification. See the Appendix.

${ }^{3}$ We do not use an immortal link and we do not assume that the length process is at stationarity. Our techniques can also be applied to the TKF91 model without much modifications. We leave the details to the reader.
} 
- let $I_{0}, I_{1}, \ldots, I_{K_{v}}$ be exponential random variables with rate $\lambda_{e}, D_{1}, \ldots, D_{K_{v}}$ exponential random variables with rate $\mu_{e}$, and $M_{1}, \ldots, M_{K_{v}}$ exponential random variables with rate $\eta_{e}$; suppose that these random variables are mutually independent and let $\mathcal{T}$ be their minimum;

- if $\mathcal{T}>t_{\ell}$ break; otherwise: if $I_{j}=\mathcal{T}$, insert a new site whose value is chosen uniformly at random from $\{0,1\}$ between the sites $\sigma_{v}^{j}$ and $\sigma_{v}^{j+1}$ of $\sigma_{v},{ }^{4}$ if $D_{j}=\mathcal{T}$, delete the site $\sigma_{v}^{j}$ from $\sigma_{v}$; and if $M_{j}=\mathcal{T}$, replace $\sigma_{v}^{j}$ by $1-\sigma_{v}^{j}$;

- update $\sigma_{v}$ according to these changes, and update $K_{v}$ to reflect the new sequence length; set the remaining time $t_{\ell}:=t_{\ell}-\mathcal{T}$;

In words, the evolutionary process defined above assumes that every site of the sequence $\sigma_{u}$ of the parent species is, independently from the other sites, subjected to a sequence of evolutionary events that flip its value; these events are distributed according to a Poisson point process of intensity $\eta_{e}$ in the time interval $\left[0, t_{e}\right]$. However, the site may get deleted and therefore not be inherited by the sequence of the node $v$; this is determined by whether an exponential random variable of rate $\mu_{e}$ is smaller than $t_{e}$. While each site of the parental sequence $\sigma_{u}$ is subjected to this process, new sites are introduced in the space between existing sites at rate $\lambda_{e}$, and each of these sites follows a similar process for the remaining time.

Given the evolutionary process on a branch of the phylogeny, the evolutionary process on the whole phylogeny is defined as follows.

Definition 1.2 (Evolutionary Process) Suppose that every site of the sequence $\sigma_{r(T)}$ at the root of the phylogeny is chosen to be 0 or 1 uniformly at random. Recursively, if $\sigma_{u}$ is the sequence at node $u$ and $e=(u, v)$ is an edge of the phylogeny, the sequence $\sigma_{v}$ at node $v$ is obtained from the sequence $\sigma_{u}$ by an application of the evolutionary process on a branch described by Definition 1.1.

For ease of exposition, we present our proof in the special case when the substitution, insertion and deletion rates are the same on all edges of the phylogeny. We discuss the more general case in the Appendix.

Definition 1.3 (Molecular Clock Assumption) Under the molecular clock assumption, there exist $\eta$, $\mu$ and $\lambda$ such that $\eta_{e}=\eta, \mu_{e}=\mu$ and $\lambda_{e}=\lambda$, for all $e$.

Notation. In the sequel, we label the leaves of the phylogeny with the positive integers $1,2, \ldots, n$, so that $L=\{1, \ldots, n\}$, and the root $r(T)$ of the phylogeny with 0 .

\subsection{Main Result}

Statement of results. We begin with a consistency result.

Theorem 1 (Consistency) Assume that $0<t_{e}, \eta_{e}<+\infty$, for all $e \in E$. Moreover, assume that the indel rates satisfy $\lambda_{e}<\mu_{e}$ for all $e \in E$. Under these assumptions, there exists an algorithm solving the phylogenetic reconstruction problem (that is, returning the correct tree) with probability of failure approaching 0 as the sequence length at the root of the tree goes to $+\infty$.

Our main result is the following. For simplicity we work under the symmetric two-state case and assume that the Molecular Clock Assumption holds. We show in Section D that these assumptions are not necessary for our results to hold.

\footnotetext{
${ }^{4}$ Clearly, if $j=0$, then $\sigma_{v}^{j}$ is undefined and, if $j=K_{v}$, then $\sigma_{v}^{j+1}$ is undefined.
} 
Theorem 2 (Main Result: Two-State Ultrametric Case) Assume there exist constants $0<f, g<+\infty$, independent of $n$, such that all branch lengths $t_{e}, e \in E$, satisfy $f<t_{e}<g$. Moreover, assume that $\eta_{e}=\eta$, for all $e \in E$, where $\eta$ is bounded between two constants $\eta>0$ and $\bar{\eta}<+\infty$ independent of $n$, and that the indel rates satisfy $\lambda_{e}=\lambda, \mu_{e}=\mu$, for all $e \in E$, and $\lambda<\mu=O(1 / \log n)$. Under the assumptions above, there exists a polynomial-time algorithm solving the phylogenetic reconstruction problem (that is, returning the correct tree) with probability of failure $O\left(n^{-\beta^{\prime}}\right)$, if the root sequence has length $k_{r}=\operatorname{poly}_{\beta^{\prime}}(n)$.

Remark 1.4 (Branch Lengths) Our assumption that all branch lengths $t_{e}, e \in E$, satisfy $f<t_{e}<g$ is standard in the sequence-length requirement literature following the seminal work of [ESSW99a].

Remark 1.5 (Indel Rates) Under our assumptions about the branch lengths given in Theorem 2, it follows (see the proof of Theorem 2 in Section C) that the evolutionary time from the root of the tree to the leaves is $\Theta(\log n)$. This implies (see the proof of Lemma 3.2) that as long as $\lambda<\mu=O(1 / \log n)$, a constantindependent of $n$ but potentially arbitrarily small, say 1 in a 100-fraction of the sites of the root sequence "survive" all the way to the leaves of the tree with high probability. Theorem 2 implies that this constant fraction of surviving sites provides sufficient information for the phylogenetic reconstruction problem to be solvable. On the other hand, if the indel rates are significantly higher than $1 / \log n$, the sequences at the leaves of the tree may experience wild variations in length-a case which appears difficult to analyze.

Remark 1.6 (Supercritical Case) For convenience, our result is stated for the case $\mu>\lambda$ which is the most relevant in practice. Our algorithm can be extended easily to the cases $\mu<\lambda$ and $\mu=\lambda$. We leave the details to the reader.

Proof sketch. As we noted before, unlike the classical setting where the Hamming distance can be analyzed through standard concentration inequalities, proving rigorously that our approach works involves several new technical difficulties. The proof goes through the following steps:

1. Expectations. We first compute expectations of block statistics, which involve analyzing a continuoustime Markov process. We use these calculations to define an appropriate additive metric based on correlations between blocks.

2. Sequence length and site displacements. We give bounds on how much sequence lengths vary across the tree, through a moment-generating function argument. Using our bounds on the sequence length process, we bound the worst-case displacements of the sites. Namely we show that, under our assumptions, all sites move by at most $O(\sqrt{k \log k})$.

3. Sequence Partitioning. We divide each sequence in blocks of size roughly $k^{\zeta}$ for $\zeta>1 / 2$, where $k$ is the sequence length at the root. From our bounds on site displacements, it follows that the blocks roughly match across different sequences. In particular, we bound the number of homologous sites between matching blocks with high probability and show that the expected correlation between these blocks is approximately correct.

4. Concentration. Finally, we show that our estimates are concentrated. The concentration argument proceeds by conditioning on the indel process satisfying the high-probability conditions in the previous points.

The crux of our result is the proper estimation of an additive metric. With such an estimation procedure in hand, we can use a standard distance-based approach to recover the phylogeny. 
Organization. The rest of the paper is organized as follows. The evolutionary distance forming the basis of our approach is presented in Section 2. We describe our full distance estimator in Section 3 and prove its concentration in the same section. Extensions are described briefly in the Appendix.

\section{Evolutionary Distances}

In this section, we show how to define an appropriate notion of "evolutionary distance" between two species. Although such distances have been widely used in prior phylogenetic work and have been defined for a variety of models [SS03, Fel04], to our knowledge our definition is the first that applies to models with indels. We begin by reviewing the standard definition in the indel-free case and then adapt it to the presence of indels. Our estimation procedure is discussed in Section 3. Throughout, we assume $\mu>\lambda$.

\subsection{The Classical Indel-free Case}

Suppose first that $\lambda_{e}=\mu_{e}=0$ for all $e$, that is, there is no indel. In that case, the sequence length remains fixed at $k$ and the alignment problem is trivial. Underlying all distance-based approaches is the following basic definition.

Definition 2.1 (Additive Metric) A phylogeny is naturally equipped with a so-called additive metric on the leaves $\mathcal{D}: L \times L \rightarrow(0,+\infty)$ defined as follows $\forall a, b \in L, \mathcal{D}(a, b)=\sum_{e \in \mathrm{P}_{T}(a, b)} \omega_{e}$, where $\mathrm{P}_{T}(a, b)$ is the set of edges on the path between $a$ and $b$ in $T$ and where $\omega_{e}$ is a nonnegative function of the parameters on $e$-in our case, $t_{e}, \eta_{e}, \lambda_{e}$, and $\mu_{e}$. For instance, a common choice for $\omega_{e}$ would be $\omega_{e}=\eta_{e} t_{e}$ in which case $\mathcal{D}(a, b)$ is the expected number of substitutions per site between a and $b$. Often $\mathcal{D}(a, b)$ is referred to as the "evolutionary distance" between species $a$ and $b$. Additive metrics are characterized by the following four-point condition: for all $a, b, c, d \in L, \mathcal{D}(a, b)+\mathcal{D}(c, d) \leq \max \{\mathcal{D}(a, c)+\mathcal{D}(b, d), \mathcal{D}(a, d)+\mathcal{D}(b, c)\}$. Moreover, assuming $\omega_{e}>0$ for all $e \in E$, it is well-known that there exists a one-to-one correspondence between $\mathcal{D}$ and $T$ as a weigthed tree with edge weights $\left\{\omega_{e}\right\}_{e \in E}$. We will discuss algorithms for constructing $T$ from $\mathcal{D}$ in Section C. For more background on tree-based metrics, see [SSO3].

Definition 2.1 implies that phylogenies can be reconstructed by computing $\mathcal{D}(a, b)$ for all pairs of leaves $a, b \in L$. Assume we seek to estimate the evolutionary distance between species $a$ and $b$ using their respective sequences. In a first attempt, one might try the (normalized) Hamming distance between $\sigma_{a}=\left(\sigma_{a}^{1}, \ldots, \sigma_{a}^{k}\right)$ and $\sigma_{b}=\left(\sigma_{b}^{1}, \ldots, \sigma_{b}^{k}\right)$. However, the expected Hamming distance-in other words, the probability of disagreement between a site of $a$ and $b$-does not form an additive metric as defined in Definition 2.1. Instead, it is well-known that an approriate estimator is obtained by "correcting" the Hamming distance for "multiple" substitions. Denoting by $\widehat{\mathcal{H}}\left(\sigma_{a}, \sigma_{b}\right)$ the Hamming distance between $\sigma_{a}$ and $\sigma_{b}$, a Markov chain calculation shows that $\mathcal{D}(a, b)=-\frac{1}{2} \log \left(1-2 \mathbb{E}\left[\widehat{\mathcal{H}}\left(\sigma_{a}, \sigma_{b}\right)\right]\right)$, with the choice $\omega_{e}=\eta_{e} t_{e}$. See e.g. [Fel04]. In a distance-based reconstruction procedure, one first estimates $\mathcal{D}$ with

$$
\widehat{\mathcal{D}}(a, b)=-\frac{1}{2} \log \left(1-2 \widehat{\mathcal{H}}\left(\sigma_{a}, \sigma_{b}\right)\right),
$$

and then applies one of the algorithms discussed in Section $\mathrm{C}$ below. The sequence-length requirement of such a method can be derived by using concentration results for $\widehat{\mathcal{H}}$ [ESSW99a, Att99].

\subsection{Taking Indels into Account}

In the presence of indels, the estimator (1) based on the Hamming distance is difficult to apply. One has to first align the sequences, which cannot be done perfectly and causes biases and correlations that are hard to 
analyze. Alternatively, one could try a different string distance such as edit distance. However, computing the expectation of edit distance under indel models appears difficult.

We use a different approach involving correlations between state frequencies. We will eventually apply the estimator to large sub-blocks of the sequences (see Section 3), but we first describe it for the full sequence for clarity. For a node $u$, let $K_{u}$ be the (random) length of the sequence at $u$ and $Z_{u}$, the number of 0 's in the sequence at $u$. Then, our distance estimator is $\widehat{\mathcal{D}}(a, b)=\left(Z_{a}-\frac{1}{2} K_{a}\right)\left(Z_{b}-\frac{1}{2} K_{b}\right)$. We now analyze the expectation of this quantity. For $u \in V$, we let $\Delta_{u}=Z_{u}-\frac{1}{2} K_{u}$, be the deviation of $Z_{u}$ from its expected value (conditioned on the sequence length).

Single channel. Suppose $T$ is made of a single edge from the root $r$ to a leaf $a$ with parameters $t, \eta, \lambda, \mu$. Assume first that the original sequence length is $k_{r}=1$. Let $K_{a}$ be the length of the sequence at $a$. Then, by Markov chain calculations [AN72, Section III.5], it can be shown that the moment-generating function of $K_{a}$ is

$$
F(s, t) \equiv \mathbb{E}\left[s^{K_{a}}\right]=\frac{\mu(s-1)-e^{(\mu-\lambda) t}(\lambda s-\mu)}{\lambda(s-1)-e^{(\mu-\lambda) t}(\lambda s-\mu)} .
$$

By differentiating $F(s, t)$ we can derive

$$
\mathbb{E}\left[K_{a}\right]=e^{-(\mu-\lambda) t},
$$

and

$$
\operatorname{Var}\left[K_{a}\right]=\frac{\mu+\lambda}{\mu-\lambda}\left[e^{-(\mu-\lambda) t}-e^{-2(\mu-\lambda) t}\right] .
$$

Let $K_{a}^{*}$ be the number of "new" sites at $a$, that is, excluding the original site if it survived. (We ignore the substitutions for the time being.) The probability that the original site survives is $e^{-\mu t}$. Then,

$$
\mathbb{E}\left[K_{a}^{*}\right]=\mathbb{E}\left[K_{a}-\mathbb{1}\{\text { original site survives }\}\right]=e^{-(\mu-\lambda) t}-e^{-\mu t},
$$

by linearity of expectation.

We now take into account the substitutions. Assume that the original sequence length at $r$ is a random variable $K_{r}$ and that the sequence at $r$ is i.i.d. uniform. Denote by $Z_{r}$ the number of 0 's at $r$. The probability that a site in $r$ that is still surviving in $a$ has flipped its value is

$$
p=\mathbb{P}[\text { state flips odd number of times in time } t]=\sum_{j=0}^{+\infty} e^{-\eta t} \frac{(\eta t)^{2 j+1}}{(2 j+1) !}=e^{-\eta t} \sinh \eta t=\frac{1-e^{-2 \eta t}}{2} .
$$

Also, note that a new site created along the path between $r$ and $a$ has equal chance of being 0 or 1 at the end of the path. Then, we have:

Lemma 2.2 (Single Channel: Expected Deviation) The following holds: $\mathbb{E}\left[\Delta_{a} \mid K_{r}, Z_{r}\right]=e^{-(2 \eta+\mu) t} \Delta_{r}$.

Fork channel. Consider now a "fork" tree, that is, a root $r$ from which emanates a single edge $e_{u}=(r, u)$ which in turn branches into two edges $e_{a}=(u, a)$ and $e_{b}=(u, b)$. For $x=a, b, u$, we denote the parameters of edge $e_{x}$ by $t_{x}, \lambda_{x}, \mu_{x}, \eta_{x}$. Our goal is to compute $\mathbb{E}[\widehat{\mathcal{D}}(a, b)]$ assuming that the sequence length at the root is $k_{r}$. We use (7), the Markov property and the fact that $Z_{u}$ conditioned on $K_{u}$ is a binomial with parameters $1 / 2$ and $K_{u}$. We get:

Lemma 2.3 (Fork Channel: Expected Distance) The following holds:

$$
\mathbb{E}[\widehat{\mathcal{D}}(a, b)]=e^{-\left(2 \eta_{a}+\mu_{a}\right) t_{a}} e^{-\left(2 \eta_{b}+\mu_{b}\right) t_{b}} e^{-\left(\mu_{u}-\lambda_{u}\right) t_{u}} \frac{k_{r}}{4} .
$$


Molecular clock. We specialize the previous result to the Molecular Clock Assumption. That is, we assume, for $x=a, b, u$, that $\lambda_{x}=\lambda, \mu_{x}=\mu$, and $\eta_{x}=\eta$. Note that by construction $t_{a}=t_{b}$ (assuming species $a$ and $b$ are contemporary). We denote $t=t_{a}$ and $\bar{t}=t_{u}+t_{a}$. Denoting $\kappa=\frac{k_{r} e^{-(\mu-\lambda) \bar{t}}}{4}$, we then get:

Lemma 2.4 (Molecular Clock: Expected Distance) The following holds: $\mathbb{E}[\widehat{\mathcal{D}}(a, b)]=e^{-(4 \eta+\mu+\lambda) t} \kappa$.

Letting $\beta=4 \eta+\mu+\lambda$, we get that $-2 \log \mathbb{E}\left[\kappa^{-1} \widehat{\mathcal{D}}(a, b)\right]=2 \beta t$, which is the evolutionary distance between $a$ and $b$ with the choice $\omega_{e}=\beta t_{e}$. Therefore, we define the following estimator $\widehat{\mathcal{D}}^{*}(a, b)=$ $-2 \log \kappa^{-1} \widehat{\mathcal{D}}(a, b)$, where we assume that $\mu, \lambda, \eta, \kappa$ are known.

\section{Distance Computation}

We now show how to estimate the evolutionary distance between two species by decomposing the sequences into large blocks which serve as roughly independent samples. We use the following notation: $M_{t}=$ $e^{-(\mu-\lambda) t}, D_{t}=e^{-\mu t}, \delta=\mu-\lambda, \phi=\mu+\lambda$, and $\Gamma_{t}=\lambda \delta^{-1}\left(1-M_{t}\right)$.

Remark 3.1 Under our main assumptions, the quantities $M_{t}, D_{t}$, and $\Gamma_{t}$ are essentially constants, that is, $O(1)$. See Section $C$. We use this fact throughout this section.

\subsection{Concentration of the Indel Process}

Sequence length. We first show that the sequence length is concentrated. Let $T$ be single channel consisting of edge $e=(r, a)$. Let $k_{r}$ be the length at $r$.

Lemma 3.2 (Single Channel: Large Deviations of Sequence Length) For all $\gamma>0$, there exists a constant $c=c\left(M_{t}, \Gamma_{t} ; \gamma\right)>0$, such that, for all $\widehat{k}_{r} \geq k_{r}$, with probability at least $1-\widehat{k}_{r}^{-\gamma}$,

$$
K_{a}=k_{r} M_{t} \pm c \sqrt{\widehat{k}_{r} \log \widehat{k}_{r}}
$$

Correlated sites. Now let $T$ be the fork channel consisting of nodes $r, u, a$ and $b$ as in Figure A.1. Assume that $a$ and $b$ are contemporary, call $t$ the time separating them from $u$, and denote by $S_{a b}$ the number of sites in $a$ and $b$ that are jointly surviving from $u$. These are the sites that produce correlation between the sequences at $a$ and $b$. All other sites are essentially noise. We bound the large deviations of $S_{a b}$.

Lemma 3.3 (Fork Channel: Large Devations of Jointly Surviving Sites) Condition on the sequence length at $u$ being $k_{u}$. Then, for all $\gamma>0$, there exists a constant $c=c\left(D_{t} ; \gamma\right)>0$, such that, for all $\widehat{k}_{u} \geq k_{u}$, with probability at least $1-\widehat{k}_{u}^{-\gamma}$,

$$
S_{a b}=k_{u} D_{t}^{2} \pm c \sqrt{\widehat{k}_{u} \log \widehat{k}_{u}}
$$

\subsection{Sequence Partitioning}

From Lemma 3.2, it follows that the sites of the root sequence (or of an internal sequence) remain fairly close to their expected position at the leaves. We take advantage of this fact by dividing each sequence into blocks of size asymptotically larger than the typical displacement implied by Lemma 3.2. As a result, matching blocks in different sequences share a significant fraction of sites. Moreover, distinct blocks are roughly independent. We estimate the evolutionary distance between two leaves by comparing the site frequencies in matching blocks. This requires some care as we show next.

Consider the fork channel. We seek to estimate the evolutionary distance $\widehat{\mathcal{D}}(a, b)$ between $a$ and $b$ (normalized by the sequence length at $u$ ). 
Partitioning the leaf sequences. Let $k_{0}$ be some deterministic length (to be determined), and consider the first $k_{0}$ sites in the sequences $\sigma_{a}$ and $\sigma_{b}$ at the nodes $a$ and $b$ respectively. If the sequence at $a$ or $b$ has length smaller than $k_{0}$, we declare that our distance estimate $\widetilde{\mathcal{D}}(a, b)$ (see below) is $+\infty$.

We divide the leaf sequences into $L$ blocks of length $\ell$ where $\ell=\left\lceil k_{0}^{\zeta}\right\rceil$, for some $\frac{1}{2}<\zeta<1$ to be determined later, and $L=\left\lfloor k_{0} / \ell\right\rfloor$. We let $k_{0}^{\prime}=\ell L$. For all $i=1, \ldots, L$, we define the $i$-th block $\sigma_{a, i}$ of $a$ to be the subsequence of $\sigma_{a}$ ranging from position $(i-1) \ell+1$ to position $i \ell$. We let $Z_{a, i}$ be the number of zeros inside $\sigma_{a, i}$ and define the block deviations $\Delta_{a, i}=Z_{a, i}-\frac{\ell}{2}$, for all $i=1, \ldots, L$. And similarly for the sequence at $b$.

Using the above notation we define our distance estimator next. Assume that $L$ is even. Otherwise, we can just drop the last block in the above partition. Our estimator is the following:

$$
\widetilde{\mathcal{D}}(a, b)=\frac{2}{L} \sum_{j=0}^{L / 2-1} \Delta_{a, 2 j+1} \Delta_{b, 2 j+1} .
$$

Notice that in our summation above we skipped every other block in our sequence partition to avoid overlapping sites and hence decrease potential correlations between the terms in the estimator. In the rest of this section, we analyze the properties of $\widetilde{\mathcal{D}}(a, b)$. To do this it is helpful to consider the sequence at $u$ and the events that happened in the channels defined by the edges $(u, a)$ and $(u, b)$.

Partitioning the ancestral sequence. Let us choose $\ell_{u}$ to be the largest integer satisfying

$$
\ell_{u} M_{t} \leq \ell
$$

Suppose that the sequence $\sigma_{u}$ at node $u$ is not shorter than $k_{u}^{\prime}=(L-1) \ell_{u}$, and define the $i$-th ancestral block $\sigma_{u, i}$ of $u$ to be the subsequence of $\sigma_{u}$ ranging from position $(i-1) \ell_{u}+1$ to position $i \ell_{u}$, for all $i \leq L-1$. Given Lemma 3.2, the choice of $\ell_{u}$ in (5) is such that the blocks of $u$ and the corresponding blocks at $a$ and $b$ roughly align.

In order to use the expected evolutionary distance as computed in Lemma 2.4, we define an "interior" ancestral block which is guaranteed with high probability to remain entirely "inside" the corresponding leaf block. Let $\delta_{u}=\left\lceil L+\frac{c}{M_{t}} \sqrt{k_{u}^{\prime} \log k_{u}^{\prime}}\right\rceil$, where $c$ is the maximum of the constants defined in the proof of Lemma 3.2 and Lemma 3.3. (The $L=o\left(\sqrt{k_{0}}\right)$ in $\delta_{u}$ is needed only when (5) is a strict inequality. See the proof of Lemma 3.4.) We define the $i$-th (ancestral) interior block $\sigma_{u, i}^{\prime}$ of $u$ to be the subsequence of $\sigma_{u, i}$ ranging from position $(i-1) \ell_{u}+\delta_{u}$ of $\sigma_{u}$ to position $i \ell_{u}-\delta_{u}$. Notice that $\delta_{u} \sim \sqrt{k_{0} \log k_{0}}$, while $\ell_{u} \sim k_{0}^{\zeta}$. Therefore, for $k_{0}>k_{0}^{*}$, where $k_{0}^{*}=k_{0}^{*}(\mu, \lambda, t, \gamma)>0$ is sufficiently large, $(i-1) \ell_{u}+\delta_{u} \ll i \ell_{u}-\delta_{u}$ so that the sequence $\sigma_{u, i}^{\prime}$ is well-defined.

Also, for all $i=1, \ldots, L-1$, we define $x_{a, i}^{\prime}, y_{a, i}^{\prime}$ to be the position of the leftmost, respectively rightmost, site in the sequence $\sigma_{a}$ descending from the site at position $(i-1) \ell_{u}+\delta_{u}$, respectively $i \ell_{u}-\delta_{u}$ of $\sigma_{u}$. Similarly we define $x_{b, i}^{\prime}$ and $y_{b, i}^{\prime}$. Given this notation, we define the following "good" event

$$
\mathcal{E}_{1}^{\prime}=\left\{\forall i \leq L-1:(i-1) \ell<x_{a, i}^{\prime}, x_{b, i}^{\prime}<(i-1) \ell+2 M_{t} \delta_{u}, i \ell-2 M_{t} \delta_{u}<y_{a, i}^{\prime}, y_{b, i}^{\prime}<i \ell\right\} .
$$

Intuitively, when the event $\mathcal{E}_{1}^{\prime}$ holds, all descendants of the interior block $\sigma_{u, i}^{\prime}$ are located inside the blocks $\sigma_{a, i}$ and $\sigma_{b, i}$ respectively (and they do not shrink much).

To argue about block independence, we also define the exterior block $\sigma_{u, i}^{\prime \prime}$ of $u$ to be the subsequence of $\sigma_{u, i}$ ranging from position $(i-1) \ell_{u}-\delta_{u}$ of $\sigma_{u}$ to position $i \ell_{u}+\delta_{u}$ with corresponding positions $x_{a, i}^{\prime \prime}, y_{a, i}^{\prime \prime}$, $x_{b, i}^{\prime \prime}$ and $y_{b, i}^{\prime \prime}$ and good event $\mathcal{E}_{1}^{\prime \prime}$ defined similarly as above.

We show that the event $\mathcal{E}_{1}=\mathcal{E}_{1}^{\prime} \cup \mathcal{E}_{1}^{\prime \prime}$ holds with high probability, conditioned on the sequence length $K_{u}$ at $u$ being at least $k_{u}^{\prime}$. Figure A. 2 shows the structure of the indel process in the case that the event $\mathcal{E}_{1}$ holds. 
Lemma 3.4 (Interior/Exterior Block Is Inside/Outside Leaf Block) Conditioned on the event $\left\{K_{u} \geq k_{u}^{\prime}\right\}$, we have

$$
\mathbb{P}\left[\mathcal{E}_{1}\right] \geq 1-16 L\left(\frac{1}{k_{u}^{\prime}}\right)^{\gamma} .
$$

Block correlation. Let $S_{a b, i}$ be the number of common sites in the blocks $\sigma_{a, i}$ and $\sigma_{b, i}$ that are jointly surviving from $u$. Similarly we define $S_{a b, i}^{\prime}$ and $S_{a b, i}^{\prime \prime}$ where, for $\xi=a, b, \sigma_{\xi, i}^{\prime}\left(\sigma_{\xi, i}^{\prime \prime}\right)$ denotes the subsequence of $\sigma_{\xi}$ ranging from position $x_{\xi, i}^{\prime}\left(x_{\xi, i}^{\prime \prime}\right)$ to position $y_{\xi, i}^{\prime}\left(y_{\xi, i}^{\prime \prime}\right)$. We define a good event for $S_{a b, i}$ as

$$
\mathcal{E}_{2}=\left\{\forall i \leq L-1: \ell_{u} D_{t}^{2}-3 M_{t} \delta_{u} \leq S_{a b, i} \leq \ell_{u} D_{t}^{2}+3 M_{t} \delta_{u}\right\} .
$$

Lemma 3.5 (Jointly Surviving Sites in Blocks) Conditioned on the event $\left\{K_{u} \geq k_{u}^{\prime}\right\}$, we have

$$
\mathbb{P}\left[\mathcal{E}_{2}\right] \geq 1-18 L\left(\frac{1}{k_{u}^{\prime}}\right)^{\gamma}
$$

\subsection{Estimation Guarantees}

We are now ready to analyze the behavior of our estimate $\widetilde{\mathcal{D}}(a, b)$. In this subsection we compute the expectation and variance of $\widetilde{\mathcal{D}}(a, b)$. We denote by $\mathcal{I}$ a realization of the indel process (but not of the substitution process) on the paths between $u$ and $a, b$. We denote by $\mathcal{E}$ the event such that $\left\{K_{u} \geq k_{u}^{\prime}\right\}, \mathcal{E}_{1}$, and $\mathcal{E}_{2}$ are satisfied. Suppose that $k_{0}>k_{0}^{*}$.

Lemma 3.6 (Block Independence) Conditioning on $\mathcal{I}$ and $\mathcal{E}$, the variables $\left\{\Delta_{a, 2 j+1} \Delta_{b, 2 j+1}\right\}_{j=1}^{L / 2-1}$ are mutually independent.

Lemma 3.7 (Expected Correlation under Good Event) We have

$$
\mathbb{E}\left[\Delta_{a, i} \Delta_{b, i} \mid \mathcal{I}, \mathcal{E}\right]=\frac{1}{4} e^{-4 \eta t} e^{-2 \mu t} \ell_{u} \pm O\left(\sqrt{k_{0} \log k_{0}}\right) .
$$

Lemma 3.8 (Variance under Good Event) We have $\operatorname{Var}\left[\Delta_{a, i} \Delta_{b, i} \mid \mathcal{I}, \mathcal{E}\right] \leq \frac{3}{16} \ell^{2}$.

Lemma 3.9 (Distance Estimate) We have

$$
\mathbb{E}[\widetilde{\mathcal{D}}(a, b) \mid \mathcal{I}, \mathcal{E}]=\frac{1}{4} e^{-(4 \eta+\mu+\lambda) t} \ell \pm O\left(\sqrt{k_{0} \log k_{0}}\right),
$$

and $\operatorname{Var}[\widetilde{\mathcal{D}}(a, b) \mid \mathcal{I}, \mathcal{E}] \leq \frac{3}{8} \frac{1}{\left\lfloor k_{0}^{1-\zeta}\right\rfloor} \ell^{2}$. In particular, the standard deviation $\operatorname{STD}[\widetilde{\mathcal{D}}(a, b) \mid \mathcal{I}, \mathcal{E}]=$ $O\left(k_{0}^{\frac{3 \zeta-1}{2}}\right)=o\left(\sqrt{k_{0}}\right)$, for $\zeta>1 / 2$ small enough.

\subsection{Concentration}

We now show that our distance estimate is concentrated. For notational convenience, we denote by $\mathbb{P}_{u}^{*}$ the probability measure induced by conditioning on the event $\left\{K_{u} \geq k_{u}^{\prime}\right\}$. Recall that the event $\mathcal{E}$ is contained in $\left\{K_{u} \geq k_{u}^{\prime}\right\}$.

Lemma 3.10 (Concentration of Distance Estimate) Let $\alpha>0$ be such that $\zeta-\alpha>1 / 2$, and $\beta=$ $1-\zeta-2 \alpha>0$, for $\zeta>1 / 2$ small enough. Then for $k_{0}$ large enough

$$
\mathbb{P}_{u}^{*}\left[\left|\frac{4}{\ell} \widetilde{\mathcal{D}}(a, b)-e^{-(4 \eta+\mu+\lambda) t}\right|>\frac{1}{k_{0}^{\alpha}}\right] \leq O\left(\frac{1}{k_{0}^{\beta}}\right) .
$$

The proofs of Theorems 1 and 2 can be found in Section C. 


\section{References}

[ADHR09] A. Andoni, C. Daskalakis, A. Hassidim, and S. Roch. Trace reconstruction on a tree. Preprint, 2009.

[AN72] K. B. Athreya and P. E. Ney. Branching processes. Springer-Verlag, New York, 1972. Die Grundlehren der mathematischen Wissenschaften, Band 196.

[Att99] K. Atteson. The performance of neighbor-joining methods of phylogenetic reconstruction. Algorithmica, 25(2-3):251-278, 1999.

[CK01] Miklós Csurös and Ming-Yang Kao. Provably fast and accurate recovery of evolutionary trees through harmonic greedy triplets. SIAM Journal on Computing, 31(1):306-322, 2001.

[Csu02] M. Csurös. Fast recovery of evolutionary trees with thousands of nodes. J. Comput. Biol., 9(2):277-97, 2002.

$\left[\mathrm{DHJ}^{+}\right.$06] Constantinos Daskalakis, Cameron Hill, Alexander Jaffe, Radu Mihaescu, Elchanan Mossel, and Satish Rao. Maximal accurate forests from distance matrices. In RECOMB, pages 281295, 2006.

[DMR06] Constantinos Daskalakis, Elchanan Mossel, and Sébastien Roch. Optimal phylogenetic reconstruction. In STOC'06: Proceedings of the 38th Annual ACM Symposium on Theory of Computing, pages 159-168, New York, 2006. ACM.

[DMR09] Constantinos Daskalakis, Elchanan Mossel, and Sébastien Roch. Phylogenies without branch bounds: Contracting the short, pruning the deep. In RECOMB, pages 451-465, 2009.

[Edg04] Robert C. Edgar. MUSCLE: multiple sequence alignment with high accuracy and high throughput. Nucl. Acids Res., 32(5):1792-1797, 2004.

[Eli06] Isaac Elias. Settling the intractability of multiple alignment. Journal of Computational Biology, 13(7):1323-1339, 2006. PMID: 17037961.

[ESSW99a] P. L. Erdös, M. A. Steel, L. A. Székely, and T. A. Warnow. A few logs suffice to build (almost) all trees (part 1). Random Struct. Algor., 14(2):153-184, 1999.

[ESSW99b] P. L. Erdös, M. A. Steel, L. A. Székely, and T. A. Warnow. A few logs suffice to build (almost) all trees (part 2). Theor. Comput. Sci., 221:77-118, 1999.

[Fel78] J. Felsenstein. Cases in which parsimony or compatibility methods will be positively misleading. Syst. Biol., pages 401-410, 1978.

[Fel04] J. Felsenstein. Inferring Phylogenies. Sinauer, New York, New York, 2004.

[GL99] D. Graur and W.-H. Li. Fundamentals of Molecular Evolution, Second Edition. Sinauer Associates, Inc., Sunderland, MA, 1999.

[GMS08] Ilan Gronau, Shlomo Moran, and Sagi Snir. Fast and reliable reconstruction of phylogenetic trees with very short edges. To appear in SODA, 2008.

[HNW99] D. H. Huson, S. H. Nettles, and T. J. Warnow. Disk-covering, a fast-converging method for phylogenetic tree reconstruction. J. Comput. Biol., 6(3-4), 1999. 
[HS88] D. G. Higgins and P. M. Sharp. Clustal: a package for performing multiple sequence alignment on a microcomputer. Gene, 73(1):237-244, 1988.

[KMKM02] Kazutaka Katoh, Kazuharu Misawa, Kei-ichi Kuma, and Takashi Miyata. MAFFT: a novel method for rapid multiple sequence alignment based on fast Fourier transform. Nucl. Acids Res., 30(14):3059-3066, 2002.

[KT81] Samuel Karlin and Howard M. Taylor. A second course in stochastic processes. Academic Press Inc. [Harcourt Brace Jovanovich Publishers], New York, 1981.

[KZZ03] Valerie King, Li Zhang, and Yunhong Zhou. On the complexity of distance-based evolutionary tree reconstruction. In SODA '03: Proceedings of the fourteenth annual ACM-SIAM symposium on Discrete algorithms, pages 444-453, Philadelphia, PA, USA, 2003. Society for Industrial and Applied Mathematics.

[LC06] Michelle R. Lacey and Joseph T. Chang. A signal-to-noise analysis of phylogeny estimation by neighbor-joining: insufficiency of polynomial length sequences. Math. Biosci., 199(2):188215, 2006.

[LG08] Ari Loytynoja and Nick Goldman. Phylogeny-Aware Gap Placement Prevents Errors in Sequence Alignment and Evolutionary Analysis. Science, 320(5883):1632-1635, 2008.

$\left[\mathrm{LRN}^{+}\right.$09] Kevin Liu, Sindhu Raghavan, Serita Nelesen, C. Randal Linder, and Tandy Warnow. Rapid and Accurate Large-Scale Coestimation of Sequence Alignments and Phylogenetic Trees. Science, 324(5934):1561-1564, 2009.

[Met03] Dirk Metzler. Statistical alignment based on fragment insertion and deletion models. Bioinformatics, 19(4):490-499, 2003.

[MLH04] I. Miklos, G. A. Lunter, and I. Holmes. A "Long Indel" Model For Evolutionary Sequence Alignment. Mol Biol Evol, 21(3):529-540, 2004.

[Mos07] E. Mossel. Distorted metrics on trees and phylogenetic forests. IEEE/ACM Trans. Comput. Bio. Bioinform., 4(1):108-116, 2007.

[MR06] Elchanan Mossel and Sébastien Roch. Learning nonsingular phylogenies and hidden Markov models. Ann. Appl. Probab., 16(2):583-614, 2006.

[RE08] Elena Rivas and Sean R. Eddy. Probabilistic phylogenetic inference with insertions and deletions. PLoS Comput Biol, 4(9):e1000172, 092008.

[Roc08] Sébastien Roch. Sequence-length requirement for distance-based phylogeny reconstruction: Breaking the polynomial barrier. In FOCS, pages 729-738, 2008.

[SR06] Marc A. Suchard and Benjamin D. Redelings. BAli-Phy: simultaneous Bayesian inference of alignment and phylogeny. Bioinformatics, 22(16):2047-2048, 2006.

[SS99] Michael A. Steel and László A. Székely. Inverting random functions. Ann. Comb., 3(1):103113, 1999. Combinatorics and biology (Los Alamos, NM, 1998).

[SS02] M. A. Steel and L. A. Székely. Inverting random functions. II. Explicit bounds for discrete maximum likelihood estimation, with applications. SIAM J. Discrete Math., 15(4):562-575 (electronic), 2002. 
[SS03] C. Semple and M. Steel. Phylogenetics, volume 22 of Mathematics and its Applications series. Oxford University Press, 2003.

[Tha06] Bhalchandra D. Thatte. Invertibility of the TKF model of sequence evolution. Math. Biosci., 200(1):58-75, 2006.

[TKF91] Jeffrey L. Thorne, Hirohisa Kishino, and Joseph Felsenstein. An evolutionary model for maximum likelihood alignment of dna sequences. Journal of Molecular Evolution, 33(2):114-124, 1991.

[TKF92] Jeffrey L. Thorne, Hirohisa Kishino, and Joseph Felsenstein. Inching toward reality: An improved likelihood model of sequence evolution. Journal of Molecular Evolution, 34(1):3-16, 1992.

[WJ94] Lusheng Wang and Tao Jiang. On the complexity of multiple sequence alignment. Journal of Computational Biology, 1(4):337-348, 1994.

[WSH08] Karen M. Wong, Marc A. Suchard, and John P. Huelsenbeck. Alignment Uncertainty and Genomic Analysis. Science, 319(5862):473-476, 2008. 


\section{A Figures}

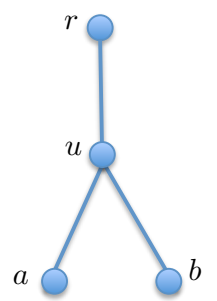

Figure A.1: The Fork Channel

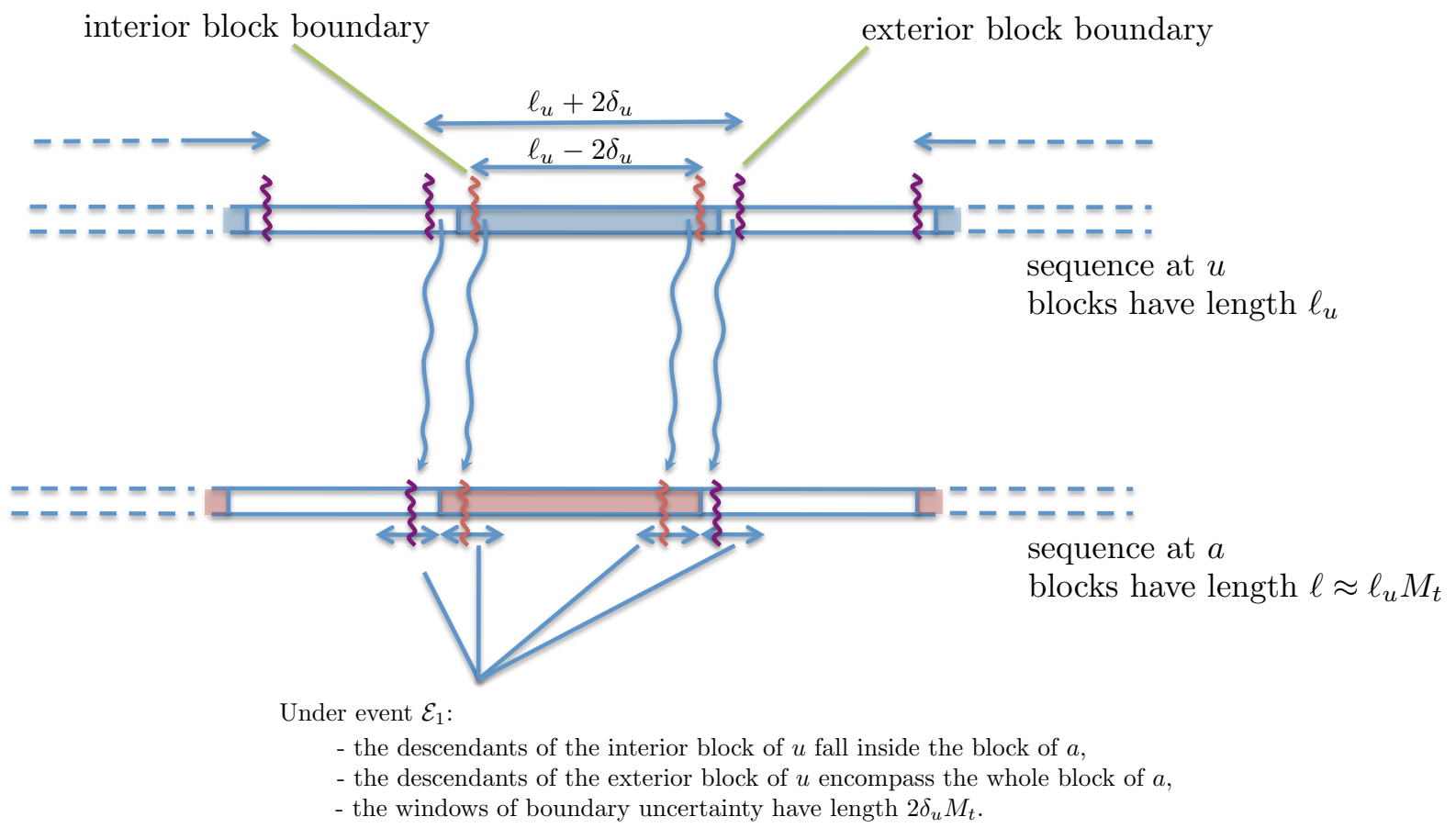

Figure A.2: Under the event $\mathcal{E}_{1}$ the descendants of the interior blocks of $\sigma_{u}$ fall inside the corresponding blocks of $\sigma_{a}$; the descendants of the exterior blocks of $\sigma_{u}$ contain all surviving sites inside the corresponding blocks of $\sigma_{a}$; the windows of uncertainty have length $2 M_{t} \delta_{u}$. 


\section{B Omitted Proofs}

Proof of Lemma 2.2: We have

$$
\begin{aligned}
\mathbb{E}\left[\Delta_{a} \mid K_{r}, Z_{r}\right]= & \mathbb{E}\left[\left(Z_{a}-\frac{1}{2} K_{a}\right) \mid K_{r}, Z_{r}\right] \\
= & Z_{r} e^{-\mu t}(1-p)+\left(K_{r}-Z_{r}\right) e^{-\mu t} p \\
& \quad+K_{r}\left(e^{-(\mu-\lambda) t}-e^{-\mu t}\right) \frac{1}{2}-K_{r} e^{-(\mu-\lambda) t} \frac{1}{2} \\
= & Z_{r}(1-2 p) e^{-\mu t}-\frac{1}{2} K_{r}(1-2 p) e^{-\mu t} \\
= & e^{-2 \eta t} e^{-\mu t} \Delta_{r},
\end{aligned}
$$

where on the first two lines:

1. the first term is the number of original 0's surviving in state 0 ;

2. the second term is the number of original 1's surviving in state 0 ;

3. the third term is the number of new sites surviving in state 0 ;

4. the fourth term is half the sequence length at $a$ given the length at $r$.

Proof of Lemma 2.3: We have

$$
\begin{aligned}
\mathbb{E}[\widehat{\mathcal{D}}(a, b)] & =\mathbb{E}\left[\Delta_{a} \Delta_{b}\right] \\
& =\mathbb{E}\left[\mathbb{E}\left[\Delta_{a} \Delta_{b} \mid K_{u}, Z_{u}\right]\right] \\
& =\mathbb{E}\left[\mathbb{E}\left[\Delta_{a} \mid K_{u}, Z_{u}\right] \mathbb{E}\left[\Delta_{b} \mid K_{u}, Z_{u}\right]\right] \\
& =e^{-2 \eta_{a} t_{a}} e^{-\mu_{a} t_{a}} e^{-2 \eta_{b} t_{b}} e^{-\mu_{b} t_{b}} \mathbb{E}\left[\Delta_{u}^{2}\right] \\
& =e^{-2 \eta_{a} t_{a}} e^{-\mu_{a} t_{a}} e^{-2 \eta_{b} t_{b}} e^{-\mu_{b} t_{b}} \mathbb{E}\left[\mathbb{E}\left[\Delta_{u}^{2} \mid K_{u}\right]\right] \\
& =e^{-2 \eta_{a} t_{a}} e^{-\mu_{a} t_{a}} e^{-2 \eta_{b} t_{b}} e^{-\mu_{b} t_{b}} \mathbb{E}\left[\frac{K_{u}}{4}\right] \\
& =e^{-2 \eta_{a} t_{a}} e^{-\mu_{a} t_{a}} e^{-2 \eta_{b} t_{b}} e^{-\mu_{b} t_{b}} \frac{e^{-\left(\mu_{u}-\lambda_{u}\right) t_{u}} k_{r}}{4}
\end{aligned}
$$

where we used (3) and Lemma 2.2.

Remark B.1 Although we stated Lemma 3.2 for the full sequence, it will also be needed for "half-sequences" and "blocks." In particular, we use the previous lemma to track the position of sites. In that context, one should think of $k_{r}$ as the position of a site in $r$ and $K_{a}$, as its position in a. Then we can use $\widehat{k}_{r}$ for the full sequence length at $r$. See Section 3.2.

Proof of Lemma 3.2: We can think of $K_{a}$ as

$$
K_{a}=\sum_{i=1}^{k_{r}} K_{a, i}
$$

where $K_{a, i}$ is the number of sites generated by a single site of the sequence at $r$. Intuitively, $K_{a, i}$ is the number of sites that were inserted between the sites $i$ and $i+1$ of the sequence at $r$, plus the site at position 
$i$ itself if it survived. Clearly the variables $\left\{K_{a, i}\right\}_{i}$ are mutually independent. Using (3) and (4) we obtain that

$$
\mathbb{E}\left[K_{a}\right]=k_{r} M_{t}
$$

and

$$
\operatorname{Var}\left[K_{a}\right]=k_{r} M_{t}\left[1-M_{t}\right] \frac{\phi}{\delta} .
$$

For $\varepsilon>0$, by Markov's inequality we have

$$
\mathbb{P}\left[K_{a} \geq k_{r} M_{t}+k_{r} \varepsilon\right] \leq s^{-k_{r}\left(M_{t}+\varepsilon\right)} \mathbb{E}\left[s^{K_{a}}\right]=\left(s^{-\left(M_{t}+\varepsilon\right)} \mathbb{E}\left[s^{K_{a, 1}}\right]\right)^{k_{r}} .
$$

We take $s=1+C \varepsilon$ for $C>0$ to be determined. By a Taylor expansion around $\varepsilon=0$, we have

$$
\begin{gathered}
\mathbb{E}\left[s^{K_{a, 1}}\right]=1+M_{t}(C \varepsilon)+M_{t} \Gamma_{t}(C \varepsilon)^{2}+O_{M_{t}, \Gamma_{t}}\left(\varepsilon^{3}\right), \\
s^{-\left(M_{t}+\varepsilon\right)}=1-\left(M_{t}+\varepsilon\right)(C \varepsilon)+\frac{\left(M_{t}+\varepsilon\right)\left(M_{t}+\varepsilon+1\right)}{2}(C \varepsilon)^{2}+O_{M_{t}}\left(\varepsilon^{3}\right),
\end{gathered}
$$

and

$$
\begin{aligned}
s^{-\left(M_{t}+\varepsilon\right)} \mathbb{E}\left[s^{K_{a, 1}}\right]=1 & -\varepsilon(C \varepsilon)+M_{t} \Gamma_{t}(C \varepsilon)^{2}+\frac{\left(M_{t}+\varepsilon\right)\left(M_{t}+\varepsilon+1\right)}{2}(C \varepsilon)^{2} \\
& -\left(M_{t}+\varepsilon\right) M_{t}(C \varepsilon)^{2}+O_{M_{t}, \Gamma_{t}}\left(\varepsilon^{3}\right) .
\end{aligned}
$$

Note that the second term on the right hand side depends on $C$ whereas the remaining terms depend on $C^{2}$. Taking $C=C\left(M_{t}, \Gamma_{t} ; \gamma\right)>0$ small enough and $c=c\left(M_{t}, \Gamma_{t} ; \gamma\right)>0$ large enough we get for

$$
\varepsilon=c \sqrt{\frac{\log \widehat{k}_{r}}{k_{r}}},
$$

that

$$
\begin{aligned}
\mathbb{P}\left[K_{a} \geq k_{r} M_{t}+c \sqrt{\widehat{k}_{r} \log \widehat{k}_{r}}\right] & \leq \mathbb{P}\left[K_{a} \geq k_{r} M_{t}+k_{r} \varepsilon\right] \\
& \leq \widehat{k}_{r}^{-\gamma}
\end{aligned}
$$

as $k_{r}$ goes to $+\infty$.

A similar inequality holds for the other direction.

Proof of Lemma 3.3: Each site in $u$ survives in $a$ with probability $D_{t}$. The same holds for $b$ independently. The result then follows from Azuma's inequality.

Proof of Lemma 3.4: It follows from Lemma 3.2 that the leftmost descendant of the site at position $(i-$ 1) $\ell_{u}+\delta_{u}$ of $\sigma_{u}$ is located inside the sequence of node $a$ at position at least

$$
\begin{aligned}
M_{t}\left((i-1) \ell_{u}+\delta_{u}\right)-c \sqrt{k_{u}^{\prime} \log k_{u}^{\prime}} & >M_{t}\left((i-1) \ell_{u}+L\right) \\
& >(i-1) \ell
\end{aligned}
$$

with probability $\geq 1-\left(\frac{1}{k_{u}^{\prime}}\right)^{\gamma}$. The other bounds follow similarly. Taking a union bound over all $i$ 's establishes the result. 
Proof of Lemma 3.5: We bound

$$
\mathbb{P}\left[\mathcal{E}_{2}^{c}\right]=\mathbb{P}\left[\mathcal{E}_{2}^{c} \cap \mathcal{E}_{1}\right]+\mathbb{P}\left[\mathcal{E}_{2}^{c} \cap \mathcal{E}_{1}^{c}\right] \leq \mathbb{P}\left[\mathcal{E}_{2}^{c} \cap \mathcal{E}_{1}\right]+\mathbb{P}\left[\mathcal{E}_{1}^{c}\right] \leq \mathbb{P}\left[\mathcal{E}_{2}^{c} \cap \mathcal{E}_{1}\right]+16 L\left(\frac{1}{k_{u}^{\prime}}\right)^{\gamma} .
$$

By construction, under $\mathcal{E}_{1}$ we have $S_{a b, i}^{\prime} \leq S_{a b, i} \leq S_{a b, i}^{\prime \prime}$ so that

$$
\begin{aligned}
\mathbb{P}\left[\mathcal{E}_{2}^{c} \cap \mathcal{E}_{1}\right] \leq \mathbb{P}[ & \left.\exists i, S_{a b, i}^{\prime} \leq \ell_{u} D_{t}^{2}-3 M_{t} \delta_{u}\right] \\
& +\mathbb{P}\left[\exists i, S_{a b, i}^{\prime \prime} \geq \ell_{u} D_{t}^{2}+3 M_{t} \delta_{u}\right] \\
\leq \mathbb{P}[ & \left.\exists i, S_{a b, i}^{\prime} \leq\left(\ell_{u}-2 \delta_{u}+1\right) D_{t}^{2}-c \sqrt{k_{u}^{\prime} \log k_{u}^{\prime}}\right] \\
& \quad+\mathbb{P}\left[\exists i, S_{a b, i}^{\prime \prime} \geq\left(\ell_{u}+2 \delta_{u}+1\right) D_{t}^{2}+c \sqrt{k_{u}^{\prime} \log k_{u}^{\prime}}\right] \\
\leq & 2 L\left(\frac{1}{k_{u}^{\prime}}\right)^{\gamma},
\end{aligned}
$$

by Lemma 3.3, where we also used the fact that $D_{t}^{2} \leq M_{t}$.

Proof of Lemma 3.6: Observe that when $K_{u} \geq k_{u}^{\prime}$ the ancestral blocks $\sigma_{u, i}$ are well defined. Assuming that $k_{0}>k_{0}^{*}$, the interior blocks $\sigma_{u, i}^{\prime}$ are also well defined and disjoint. Hence, for a fixed $\mathcal{I}$ under $\mathcal{E}$, for all $i \leq L-1$, both $\Delta_{a, i}$ and $\Delta_{b, i}$ depend on the subsequence of $\sigma_{u}$ ranging from position $(i-1) \ell_{u}-\delta_{u}+1$ to position $i \ell_{u}+\delta_{u}-1$. In this case, for $j \in\{1, \ldots, L / 2-1\}$, different $\Delta_{a, 2 j+1} \Delta_{b, 2 j+1} \mathrm{~s}$ are functions of different subsequences of $\sigma_{u}$. Observe that, since the root sequence is i.i.d. uniform and the insertions above $u$ are also i.i.d. uniform, the state of every site in $\sigma_{u}$ is uniform and independent from the other sites. It follows from the above observations that $\left\{\Delta_{a, 2 j+1} \Delta_{b, 2 j+1}\right\}_{j=1}^{L / 2-1}$ are mutually independent.

Proof of Lemma 3.7: Let $\Delta_{a, i}^{\mathrm{S}}$ be the contribution to $\Delta_{a, i}$ from those common sites between $a$ and $b$ that are jointly surviving from $u$. Let $\Delta_{a, i}^{\mathrm{NS}}=\Delta_{a, i}-\Delta_{a, i}^{\mathrm{S}}$. And similarly for $b$. Then

$$
\begin{aligned}
\mathbb{E}\left[\Delta_{a, i} \Delta_{b, i} \mid \mathcal{I}, \mathcal{E}\right] & =\mathbb{E}\left[\left(\Delta_{a, i}^{\mathrm{S}}+\Delta_{a, i}^{\mathrm{NS}}\right)\left(\Delta_{b, i}^{\mathrm{S}}+\Delta_{b, i}^{\mathrm{NS}}\right) \mid \mathcal{I}, \mathcal{E}\right] \\
& =\mathbb{E}\left[\Delta_{a, i}^{\mathrm{S}} \Delta_{b, i}^{\mathrm{S}} \mid \mathcal{I}, \mathcal{E}\right],
\end{aligned}
$$

since the contribution from $\Delta_{a, i}^{\mathrm{NS}}$ and $\Delta_{b, i}^{\mathrm{NS}}$ is independent and averages to 0 . Write $\Delta_{a, i}^{\mathrm{S}}$ as a sum over the jointly surviving sites, that is,

$$
\Delta_{a, i}^{\mathrm{S}}=\sum_{j=1}^{S_{a b, i}}\left(z_{a, i}^{(j)}-\frac{1}{2}\right),
$$

where $z_{a, i}^{(j)}$ is 1 if the corresponding site of $a$ is 0 . Note that the terms in parentheses have zero expectation under $\mathcal{I}$ and $\mathcal{E}$. Then,

$$
\mathbb{E}\left[\Delta_{a, i}^{\mathrm{S}} \Delta_{b, i}^{\mathrm{S}} \mid \mathcal{I}, \mathcal{E}\right]=\sum_{j=1}^{S_{a b, i}} \mathbb{E}\left[\left(z_{a, i}^{(j)}-\frac{1}{2}\right)\left(z_{b, i}^{(j)}-\frac{1}{2}\right) \mid \mathcal{I}, \mathcal{E}\right],
$$

by independence of the sites. We compute the expectation above. We have

$$
\begin{aligned}
\mathbb{E}\left[\left(z_{a, i}^{(j)}-\frac{1}{2}\right)\left(z_{b, i}^{(j)}-\frac{1}{2}\right) \mid \mathcal{I}, \mathcal{E}\right] & =\mathbb{E}\left[\left(z_{a, i}^{(j)} z_{b, i}^{(j)}-\frac{1}{2} z_{a, i}^{(j)}-\frac{1}{2} z_{b, i}^{(j)}+\frac{1}{4}\right) \mid \mathcal{I}, \mathcal{E}\right] \\
& =\mathbb{E}\left[z_{a, i}^{(j)} z_{b, i}^{(j)} \mid \mathcal{I}, \mathcal{E}\right]-\frac{1}{4} \\
& =\frac{1}{2} \cdot \frac{1+e^{-4 \eta t}}{2}-\frac{1}{4} \\
& =\frac{1}{4} e^{-4 \eta t} .
\end{aligned}
$$


Therefore

$$
\mathbb{E}\left[\Delta_{a, i}^{\mathrm{S}} \Delta_{b, i}^{\mathrm{S}} \mid \mathcal{I}, \mathcal{E}\right]=\frac{1}{4} e^{-4 \eta t} S_{a b, i}
$$

The result then follows from $\mathcal{E}_{2}$.

Proof of Lemma 3.8: By Cauchy-Schwarz, we have

$$
\begin{aligned}
\mathbb{E}\left[\Delta_{a, i}^{2} \Delta_{b, i}^{2} \mid \mathcal{I}, \mathcal{E}\right] & \leq\left(\mathbb{E}\left[\Delta_{a, i}^{4} \mid \mathcal{I}, \mathcal{E}\right] \mathbb{E}\left[\Delta_{b, i}^{4} \mid \mathcal{I}, \mathcal{E}\right]\right)^{1 / 2} \\
& =\left(\frac{1}{16}\left(3 \ell^{2}-2 \ell\right) \cdot \frac{1}{16}\left(3 \ell^{2}-2 \ell\right)\right)^{1 / 2} \\
& \leq \frac{3}{16} \ell^{2}
\end{aligned}
$$

where we used the fact that the length of the sequences $\sigma_{a, i}$ and $\sigma_{b, i}$ is deterministically $\ell$, and the number of zeros in $\sigma_{a, i}$ and $\sigma_{b, i}$ follows a Binomial distribution with $\ell$ trials and probability $1 / 2$.

Proof of Lemma 3.9: From Lemma 3.6, the $L / 2=\left\lfloor k_{0} / \ell\right\rfloor / 2$ terms in $\widetilde{\mathcal{D}}(a, b)$ are mutually independent. The proof then follows from Lemmas 3.7 and 3.8, and the definition of $\ell_{u}$.

Proof of Lemma 3.10: We use Chebyshev's inequality. We first condition on $\mathcal{I}, \mathcal{E}$. Recalling that $\ell=\left\lceil k_{0}^{\zeta}\right\rceil$, note that

$$
\begin{aligned}
& \mathbb{P}_{u}^{*} {\left[\frac{4}{\ell} \widetilde{\mathcal{D}}(a, b)>e^{-(4 \eta+\mu+\lambda) t}+\frac{1}{k_{0}^{\alpha}} \mid \mathcal{I}, \mathcal{E}\right] } \\
& \leq \mathbb{P}_{u}^{*}\left[\widetilde{\mathcal{D}}(a, b)>\frac{\ell}{4} e^{-(4 \eta+\mu+\lambda) t}+\frac{\ell}{4} \frac{1}{k_{0}^{\alpha}} \mid \mathcal{I}, \mathcal{E}\right] \\
& \leq \mathbb{P}_{u}^{*}\left[\widetilde{\mathcal{D}}(a, b)>\mathbb{E}[\widetilde{\mathcal{D}}(a, b) \mid \mathcal{I}, \mathcal{E}]-O\left(\sqrt{k_{0} \log k_{0}}\right)+\frac{\ell}{4} \frac{1}{k_{0}^{\alpha}} \mid \mathcal{I}, \mathcal{E}\right] \\
& \leq \frac{\frac{3}{8} \frac{1}{\left\lfloor k_{0}^{1-\zeta}\right\rfloor} \ell^{2}}{\left(\frac{\ell}{4} \frac{1}{k_{0}^{\alpha}}-O\left(\sqrt{k_{0} \log k_{0}}\right)\right)^{2}} \\
& \quad=O\left(\frac{1}{k_{0}^{1-\zeta-2 \alpha}}\right) .
\end{aligned}
$$

The other direction is similar. Taking expectation over $\mathcal{I}$, we have

$$
\mathbb{P}_{u}^{*}\left[\left|\frac{4}{\ell} \widetilde{\mathcal{D}}(a, b)-e^{-(4 \eta+\mu+\lambda) t}\right|>\frac{1}{k_{0}^{\alpha}} \mid \mathcal{E}\right] \leq O\left(\frac{1}{k_{0}^{\beta}}\right) .
$$

Choose $\gamma>0$ in Lemmas 3.2 and 3.3 large enough so that

$$
\gamma-(1-\zeta)>\beta
$$

Then, from Lemmas 3.4 and 3.5, we have

$$
\begin{aligned}
& \mathbb{P}_{u}^{*}\left[\left|\frac{4}{\ell} \widetilde{\mathcal{D}}(a, b)-e^{-(4 \eta+\mu+\lambda) t}\right|>\frac{1}{k_{0}^{\alpha}}\right] \\
& \quad \leq \mathbb{P}_{u}^{*}\left[\left|\frac{4}{\ell} \widetilde{\mathcal{D}}(a, b)-e^{-(4 \eta+\mu+\lambda) t}\right|>\frac{1}{k_{0}^{\alpha}} \mid \mathcal{E}\right] \mathbb{P}_{u}^{*}[\mathcal{E}]+\mathbb{P}_{u}^{*}\left[\mathcal{E}^{c}\right] \\
& \quad \leq O\left(\frac{1}{k_{0}^{\beta}}\right) .
\end{aligned}
$$




\section{Putting it All Together}

Large-scale asymptotics. We assume that all branch lengths $t_{e}, e \in E$, are bounded between two constants $f>0$ and $g<+\infty$ independent of $n$. We also assume that for all $e \in E, \eta_{e}$ is bounded between two constants $\underline{\eta}>0$ and $\bar{\eta}<+\infty$, which are also independent of $n$. Finally we assume that the indel rates satisfy $\lambda_{e}<\mu_{e} \leq \bar{\mu}=O(1 / \log n)$, for all $e \in E$.

We are ready to prove our main result in the molecular clock case. We postpone the more general case to the appendix. A last bit of notation: For a pair of leaves $a, b \in[n]$, we denote by $t_{a b}$ the time between $a$, $b$ and their most recent common ancestor. In the molecular clock case this time is well-defined, assuming that all leaves are contemporaneous.

Proof of Theorem 2: We first give a bound on the diameter of the tree. Let $h$ (resp. $H$ ) be the length of the shortest (resp. longest) path between the root and a leaf in graph distance. Because the number of leaves is $n$ we must have $2^{h} \leq n$ and $2^{H} \geq n$. Since all leaves are contemporaneous it must be that $H f \leq h g$. Combining these constraints gives that the diameter Diam satisfies

$$
2 \frac{f}{g} \log _{2} n \leq 2 h \leq \text { Diam } \leq 2 H \leq 2 \frac{g}{f} \log _{2} n .
$$

Given our bound on the diameter of the tree, it follows that the time from the root $r$ of the tree to any leaf is at most $2 \frac{g^{2}}{f} \log _{2} n$. Suppose that the length $k_{r}$ at the root of the tree satisfies $k_{r}>k_{r}^{*}=k_{r}^{*}\left(k_{0}\right)$, where $k_{r}^{*}$ is the minimum integer satisfying

$$
k_{r}^{*} \geq e^{\frac{2 g^{2}}{f} \cdot \mu \log _{2} n}\left(k_{0}+c \sqrt{k_{r}^{*} \log k_{r}^{*}}\right)=O(1) \cdot\left(k_{0}+c \sqrt{k_{r}^{*} \log k_{r}^{*}}\right),
$$

with $c$ taken to be the constant used in Lemma 3.2. Lemma 3.2 and the union bound imply then that with probability at least $1-O(n) \cdot\left(k_{r}^{*}\right)^{-\gamma}$ :

$$
\text { for all nodes } u: K_{u} \geq k_{u}^{\prime} \text {. }
$$

Lemma C.1 (Concentration of Distance Estimate) For all $\alpha^{\prime}>0, \beta^{\prime}>0$, there exists $k_{0}=\operatorname{poly}(n)$ large enough so that if the sequence length at the root is $k_{r}>k_{r}^{*}\left(k_{0}\right)$, then

$$
\mathbb{P}\left[\forall a, b \in[n],\left|\frac{4}{\ell} \widetilde{\mathcal{D}}(a, b)-e^{-(4 \eta+\mu+\lambda) t_{a b}}\right| \leq \frac{1}{n^{\alpha^{\prime}}}\right]=1-O\left(\frac{1}{n^{\beta^{\prime}}}\right) .
$$

Proof: Follows from Lemma 3.10 and our observation above that if $k_{r}>k_{r}^{*}\left(k_{0}\right)$ then with probability at least $1-O(n) \cdot\left(k_{r}^{*}\right)^{-\gamma}$, then $K_{u} \geq k_{u}^{\prime}$, for all nodes $u$.

Given our bound on the diameter of the tree, we can show that for all pairs of leaves $a, b$ and small $\varepsilon>0$ :

$$
e^{-(4 \eta+\mu+\lambda) t_{a b} \pm \varepsilon}=e^{-(4 \eta+\mu+\lambda) t_{a b}}(1 \pm O(\varepsilon)) \geq \frac{1}{n^{\alpha^{\prime \prime}}}(1 \pm O(\varepsilon)) .
$$

Therefore, choosing $\alpha^{\prime}$ large enough in Lemma C.1, we get that all distances can be estimated within a small $\varepsilon$ simultaneously with probability going to 1 .

Using the standard Buneman algorithm, we can recover the tree efficiently. See e.g. [SS03].

Constant-size case. The proof of Theorem 1 for the molecular clock case builds on the proof of Theorem 2 by treating $n$ as a constant and letting the sequence length at the root of the tree go to infinity. The extension of Theorem 1 to the general case builds on the generalization of Theorem 2.

Proof of Theorem 1:(Molecular clock case) We can restate Lemma C.1 in the following form, where the failure probability is expressed more cleanly in terms of the sequence length at the root of the tree. The proof of the lemma is essentially the same. 
Lemma C.2 (Concentration of Distance Estimate) For all $\alpha^{\prime}>0$, there exists $k_{0}^{*}=\operatorname{poly}(n)$ large enough such that if the sequence length at the root is $k_{r}>k_{r}^{*}\left(k_{0}^{*}\right)$, then

$$
\mathbb{P}\left[\forall a, b \in[n],\left|\frac{4}{\ell} \widetilde{\mathcal{D}}(a, b)-e^{-(4 \eta+\mu+\lambda) t_{a b}}\right| \leq \frac{1}{n^{\alpha^{\prime}}}\right]=1-O\left(n \cdot k_{r}^{-\gamma}\right)-O\left(n^{2} \cdot k_{r}^{-\beta}\right) .
$$

Repeating the proof of Theorem 1 in the previous subsection, it follows that the algorithm fails to reconstruct the phylogeny with probability $O\left(n \cdot k_{r}^{-\gamma}\right)+O\left(n^{2} \cdot k_{r}^{-\beta}\right)$. Letting $k_{r} \rightarrow+\infty$ concludes the proof of Theorem 1.

\section{Extensions}

GTR model. We briefly discuss how our results can be extended to GTR models. For background on GTR models, see e.g. [Fel04]. Let $Q$ be a reversible $4 \times 4$ rate matrix with stationary distribution $\pi$. Our new sequence evolution process is identical to the one described in Definition 1.1 except that the substitution process is a continuous-time Markov process with rate matrix $\eta_{e} Q$. The rate matrix $Q$ has 4 nonnegative eignevalues. For convenience, we assume that the largest negative eigenvalue is -1 . We denote by $w$ the corresponding eigenvector which we assume is normalized as follows

$$
\sum_{s \in\{\mathrm{A}, \mathrm{G}, \mathrm{C}, \mathrm{T}\}} \pi_{s} w_{s}^{2}=1 .
$$

We now perform the following transformation of the state space. For a node $u$, let $\sigma_{u}=\left(\sigma_{u}^{1}, \ldots, \sigma_{u}^{K_{u}}\right)$ be the transormed sequence at $u$ where $\sigma_{u}^{i}=w_{\mathrm{A}}$ (resp. $w_{\mathrm{G}}, w_{\mathrm{C}}, w_{\mathrm{T}}$ ) if the state at site $i$ is A (resp. G, C, T). Note that under stationarity, the expectation of the state at site $i$ is 0 by orthogonality of $\pi$ and $w$. Then, our distance estimator is

$$
\widehat{\mathcal{D}}(a, b)=\left(\sum_{i=1}^{K_{a}} \sigma_{a}^{i}\right)\left(\sum_{j=1}^{K_{b}} \sigma_{b}^{j}\right) .
$$

In particular, in the CFN case, we have $w=(+1,-1)$ and we obtain the same estimate as before up to a constant. We now analyze the expectation of this quantity. For $u \in V$, we let

$$
\Delta_{u}=\sum_{i=1}^{K_{u}} \sigma_{u}^{i}
$$

Lemma D.1 The following holds:

$$
\mathbb{E}\left[\Delta_{a} \mid \sigma_{r}\right]=e^{-(\eta+\mu) t} \Delta_{r}
$$

Remark D.2 Note that this formula is slightly different than that in Lemma 2.2 because of the normalization implied by requiring $Q$ to have second eigenvalue -1 .

Proof: The sites created after $r$ contribute 0 in expectation. Of course, so do the deleted sites. The fraction of sites that survive is $e^{-\mu t}$. Suppose site $i$ survives, then note that

$$
\mathbb{E}\left[\sigma_{a}^{i} \mid \sigma_{r}^{i}=w_{s}, i \text { survives }\right]=\left(e^{\eta t Q}\right)_{s s^{\prime}} w_{s^{\prime}}=e^{-\eta t} w_{s} .
$$

Summing over all sites of $r$ we get

$$
\mathbb{E}\left[\Delta_{a} \mid \sigma_{r}\right]=e^{-(\eta+\mu) t} \Delta_{r},
$$

as claimed. 
Consider now a "fork" tree, that is, a root $r$ from which emanates a single edge $e_{u}=(r, u)$ which in turn branches into two edges $e_{a}=(u, a)$ and $e_{b}=(u, b)$. For $x=a, b, u$, we denote the parameters of edge $e_{x}$ by $t_{x}, \lambda_{x}, \mu_{x}, \eta_{x}$. Our goal is to compute $\mathbb{E}[\widehat{\mathcal{D}}(a, b)]$ assuming that the sequence length at the root is $k$. The proof is similar to Lemma 2.3.

Lemma D.3 The following holds:

$$
\mathbb{E}[\widehat{\mathcal{D}}(a, b)]=e^{-\left(\eta_{a}+\mu_{a}\right) t_{a}} e^{-\left(\eta_{b}+\mu_{b}\right) t_{b}} e^{-\left(\mu_{u}-\lambda_{u}\right) t_{u}} k .
$$

Note that Remark D.2 also applies here.

Proof: We have

$$
\begin{aligned}
\mathbb{E}[\widehat{\mathcal{D}}(a, b)] & =\mathbb{E}\left[\Delta_{a} \Delta_{b}\right] \\
& =\mathbb{E}\left[\mathbb{E}\left[\Delta_{a} \Delta_{b} \mid \sigma_{u}\right]\right] \\
& =\mathbb{E}\left[\mathbb{E}\left[\Delta_{a} \mid \sigma_{u}\right] \mathbb{E}\left[\Delta_{b} \mid \sigma_{u}\right]\right] \\
& =e^{-\eta_{a} t_{a}} e^{-\mu_{a} t_{a}} e^{-\eta_{b} t_{b}} e^{-\mu_{b} t_{b}} \mathbb{E}\left[\Delta_{u}^{2}\right] \\
& =e^{-\eta_{a} t_{a}} e^{-\mu_{a} t_{a}} e^{-\eta_{b} t_{b}} e^{-\mu_{b} t_{b}} \mathbb{E}\left[\mathbb{E}\left[\Delta_{u}^{2} \mid K_{u}\right]\right] \\
& =e^{-\eta_{a} t_{a}} e^{-\mu_{a} t_{a}} e^{-\eta_{b} t_{b}} e^{-\mu_{b} t_{b}} \mathbb{E}\left[\operatorname{Var}\left[\Delta_{u} \mid K_{u}\right]\right] \\
& \left.=e^{-\eta_{a} t_{a}} e^{-\mu_{a} t_{a}} e^{-\eta_{b} t_{b}} e^{-\mu_{b} t_{b}} \mathbb{E}\left[K_{u} \operatorname{Var}\left[\sigma_{u}^{1}\right]\right]\right] \\
& =e^{-\eta_{a} t_{a}} e^{-\mu_{a} t_{a}} e^{-\eta_{b} t_{b}} e^{-\mu_{b} t_{b}} \mathbb{E}\left[K_{u} \mathbb{E}\left[\left(\sigma_{u}^{1}\right)^{2}\right]\right] \\
& =e^{-\eta_{a} t_{a}} e^{-\mu_{a} t_{a}} e^{-\eta_{b} t_{b}} e^{-\mu_{b} t_{b}} e^{-\left(\mu_{u}-\lambda_{u}\right) t_{u}} k,
\end{aligned}
$$

by Lemma D.1.

From the previous lemmas, it is easy to adapt the proofs above to the GTR case.

Nonclock case. Using Lemma D.3, we can get rid of the molecular clock assumption. Consider again the fork tree, but assume that each edge is in fact a path. A simple adaptation of Lemma D.3 gives the following.

Lemma D.4 The following holds:

$$
-\ln \left(\frac{\mathbb{E}[\widehat{\mathcal{D}}(a, b)]}{\sqrt{\mathbb{E}\left[K_{a}\right] \mathbb{E}\left[K_{b}\right]}}\right)=\sum_{e \in \mathrm{P}(a, b)}\left(\eta_{e}+\mu_{e} / 2+\eta_{e} / 2\right) t_{e}
$$

Note that Remark D.2 also applies here.

Proof: Note that

$$
-\ln \left(k^{-1} \mathbb{E}\left[K_{a}\right]\right)=\sum_{e \in \mathrm{P}(r, a)}\left(\mu_{e}-\lambda_{e}\right) t_{e},
$$

and similarly for $b$. A variant of Lemma D.3 gives

$$
-\ln \left(k^{-1} \mathbb{E}[\widehat{\mathcal{D}}(a, b)]\right)=\sum_{e \in \mathrm{P}(a, b)}\left(\eta_{e}+\mu_{e}\right) t_{e}+\sum_{e \in \mathrm{P}(r, u)}\left(\mu_{e}-\lambda_{e}\right) t_{e} .
$$

The result follows by subtracting the previous expressions.

The expression in Lemma D.4 provides the additive metric needed to extend our results to nonclock cases. A simple adaptation of our estimation procedure can be used to estimate this metric. We will give the details in the full version. 